\title{
Baicalein Inhibits MMPs Expression via a MAPK-Dependent Mechanism in Chondrocytes
}

\author{
Wei-Ping Chen Yan Xiong Peng-Fei Hu Jia-Peng Bao Li-Dong Wu \\ Department of Orthopedic Surgery, the Second Affiliated Hospital, School of Medicine, Zhejiang \\ University, Hangzhou, China
}

\section{Key Words}

Baicalein • Osteoarthritis • Matrix metalloproteinase $\bullet$ Chondrocytes $\bullet$ Interleukin-1 $\beta$

\begin{abstract}
Background: Baicalein is a flavonoid isolated from Scutellaria baicalensis Georgi. Here, we investigated the anti-osteoarthritic effect of baicalein in vitro and in vivo. Methods: Interleukin-1 beta (IL-1 $\beta$ )-induced chondrocytes were treated with different concentrations of baicalein, real-time PCR and ELISA were performed to detect the matrix metalloproteinases (MMPs) expression. Western blot was used to evaluate the mitogen-activated protein kinase (MAPK) expression. In experimental osteoarthritis (OA), rabbits were treated with baicalein, gross morphological and histological assessment was performed to evaluate the cartilage damage. Results: Baicalein significantly reduced the expression of MMPs in vitro and in vivo. Moreover, baicalein significantly reduced the phosphorylation of p38 and extracellular signal regulated kinase (ERK), but not of c-Jun N-terminal kinase (JNK). In addition, intra-articular injection of baicalein ameliorated the cartilage damage in a rabbit model of OA induced by anterior cruciate ligament transection (ACLT). Conclusions: The results indicate that baicalein may be considered as a potential agent for OA treatment.
\end{abstract}

Copyright $\odot 2015$ S. Karger AG, Basel

\section{Introduction}

Osteoarthritis (OA) is the most prevalent joint disorder in the elderly and often results in joint pain, stiffness and dysfunction. The disease is characterized by cartilage degradation, synovial inflammation and subchondral sclerosis, among which, cartilage

Li-Dong Wu, Ph.D. M.D., Professor,

KARGER 125
Department of Orthopedic Surgery, the Second Affiliated Hospital, School of Medicine, Zhejiang University, 88\# Jie Fang Road, Hangzhou, Zhejiang Province, 310009

(The People's Republic of China), Tel. +86-571-8778-3578, E-Mail Idwu@yahoo.com 
degradation is considered the central feature of OA. It is believed that cartilage degradation results from the homeostatic imbalance between matrix anabolism and catabolism. Matrix metalloproteinases (MMPs) and interleukin-beta (IL-1 $\beta$ ) are known to play pivotal roles in cartilage degradation and OA pathogenesis $[1,2]$. MMPs is a family of enzymes that contribute to cartilage degradation through degrading extracellular matrix (EMC) while IL-1 $\beta$ is a cytokine that contributes to cartilage degradation by inducing the expression of MMPs and other proteases $[3,4]$. Previous studies demonstrated that the inhibition of IL-1 $\beta$ or MMP expression using pharmacologic inhibitors exerted beneficial effects on OA $[5,6]$.

A number of drugs, including nonsteroidal anti-inflammatory drugs (NSAIDs), cyclooxygenase-2 inhibitors, glucosamine, steroids and hyaluronan, have been used in $\mathrm{OA}$ treatment; however, current drugs are unable to reverse cartilage damage. Therefore, it is considerable interest in discovering a novel potential agent for OA treatment, with natural drugs receiving increasing interest due to their tolerance and safety.

Baicalein is a flavonoid isolated from Scutellaria baicalensis Georgi (Huangqin)which possesses anti-inflammatory, anti-oxidative and anti-carcinogenic activities [7-10]. In additon, baicalein has been shown to attenuate MMP-1 expression in human keratinocytes [11]. Furthermore, baicalein has been reported to inhibit the proliferation of human rheumatoid arthritis fibroblast-like synoviocytes [12]. Whether baicalein possesses antiosteoarthritic properties remains unknown. In the present study, we investigated the antiosteoarthritic properties of baicalein and the related mechanisms. We demonstrated that baicalein inhibited IL-1 $\beta$-induced MMP expression via regulation of the extracellular signal regulated kinase (ERK) and p38 pathways. The results of the in vivo study showed that baicalein reduced cartilage degradation in $\mathrm{OA}$.

\section{Materials and Methods}

Reagents

Baicalein, dimethyl sulfoxide (DMSO), recombinant human IL-1 $\beta$ and 3-(4,5-dimethyl-thiazole2yl)-2,5-diphenyl tetrazolium bromide (MTT) were purchased from Sigma-Aldrich (St. Louis, MO, USA). Dulbecco's modified Eagle's medium (DMEM), penicillin, streptomycin, fetal bovine serum, 0.25\% trypsin and collagenase II were obtained from Gibco BRL (Grand Island, NY, USA). Baicalein was dissolved in DMSO for stock preparation.

\section{Chondrocytes culture}

This study was approved by the local Ethics Committee. Following patient consent, cartilage was obtained from OA patients undergoing total knee arthroplasty $(n=5$, mean age, 67.2 years; range, 6475 years). Chondrocytes were isolated from the cartilage as described previously [13]. Confluent cells were passaged at a ratio of 1:3, and third-passage cells were used.

Cells viability assay

Chondrocytes were cultured in 96-well plates $\left(5 \times 10^{3} /\right.$ well $)$ and incubated with different concentrations of baicalein for $24 \mathrm{~h}$. Cell viability was determined using the MTT assay as described previously [14].

Cell stimulation by $I L-1 \beta$ and baicalein treatment

Cells at $80 \%$ confluency were serum-starved overnight and pretreated with baicalein for $1 \mathrm{~h}$ prior to stimulation with IL-1 $(10 \mathrm{ng} / \mathrm{ml})$ for $24 \mathrm{~h}$. The cells were harvested for MMPs gene expression analysis. The culture media were collected for enzyme-linked immunosorbent assay (ELISA). In a further study, cells were pretreated with baicalein $(50 \mu \mathrm{M})$ for $24 \mathrm{~h}$, stimulated with IL-1 $\beta$ for $30 \mathrm{~min}$ and harvested for western blotting analysis.

Quantitative real-time polymerase chain reaction (PCR)

PCR was performed as reported previously [13]. In brief, total RNA was isolated using TRIzol reagent (Invitrogen, Carlsbad, CA, USA). cDNA was synthesized using the Moloney murine leukemia virus Reverse Transcriptase cDNA synthesis kit (Promega, Madison, WI, USA). The expression of messenger RNAs (mRNAs) 


\begin{tabular}{|c|c|c|}
\hline Cellular Physiology & Cell Physiol Biochem 2015;36:325-333 & \\
\hline and Biochemistry & $\begin{array}{l}\text { DOI: 10.1159/000374075 } \\
\text { Published online: May 05, } 2015\end{array}$ & $\begin{array}{l}\text { O } 2015 \text { S. Karger AG, Basel } \\
\text { www.karger.com/cpb }\end{array}$ \\
\hline
\end{tabular}

was quantified using real-time PCR, with actin as an internal control. Each gene analysis was performed in triplicate. Primer sequences of the targeted genes are listed in Table 1.

\section{Measuring MMPs by ELISA}

The levels of MMP-1, MMP-3 and MMP-13 in culture media were determined by ELISA using human MMP-1, MMP-3 and MMP-13 ELISA kits (R\&D Systems, Inc., Minneapolis, MN, USA).

\section{Western blotting analysis}

Western blotting analysis was performed as described previously [13]. Briefly, cells were washed using ice-cold phosphate-buffered saline (PBS) and the protein was extracted. The protein was transferred to polyvinylidene fluoride (PVDF) membranes and the membranes were incubated with blocking buffer followed by antibodies (ERK1/2, p-ERK1/2, P38, p-P38, c-Jun N-terminal kinase (JNK) and p-JNK (Cell Signaling Technology, Beverly, MA, USA). Finally, the filter was incubated with the electrochemiluminescence substrate and exposed to X-ray film (Kodak).

\section{Animal experiments}

New Zealand rabbits weighing 2.0-2.5 kg were used. The rabbits were purchased from the animal center of Zhejiang University and the study was approval by the Institutional Animal Care and Use Committee of Zhejiang University (Hangzhou, China). Thirty-two rabbits underwent anterior cruciate ligament transection (ACLT) in the right knee joint as described previously, and the animals were randomly assigned to four groups of eight rabbits each [15]. The rabbits received intraarticular injection of DMSO (as vehicle) or different concentration of baicalein $(5,25$, or $50 \mu \mathrm{M})$ in the right knee. Treatment was initiated on the day of the operation, and an injection was performed once weekly for 6 consecutive weeks. Another eight rabbits were used as a normal group, and received no injection. The rabbits were sacrificed and the right femoral condyles were harvested for assessment.

\section{Gross morphological changes}

Gross morphological changes were observed and graded by two independent blinded researchers according to following criteria: Grade 1: intact surface; Grade 2: minimal fibrillation; Grade 3: overt fibrillation; Grade 4: erosion [16].

\section{Histological assessment}

The samples were fixed in 10\% neutral-buffered formalin, decalcified and embedded. Sections of $5 \mu \mathrm{m}$ were cut and stained using Safranin 0 for histological assessment according to the Mankin score system in a blinded manner [17](Table 2).
Table 1. Primers of targeted genes. ${ }^{*} \mathrm{~S}=$ Sense; $\mathrm{A}=$ Antisense

\begin{tabular}{llcl}
\hline Targeted genes & Sequence(5'-3')* & $\begin{array}{l}\text { Amplicon } \\
\text { length(bp) }\end{array}$ & Accession number \\
\hline \multirow{2}{*}{ MMP-1 } & S: GGGAATAAGTACTGGGCTGTTCAG & 125 & NM_002421 \\
\multirow{2}{*}{ MMP-3 } & $\begin{array}{l}\text { A: CCTCAGAAAGAGCAGCATCGATATG } \\
\text { S: CCTCTATGGACCTCCCACAGAATC }\end{array}$ & 121 & NM_133523 \\
MMP-13 & $\begin{array}{l}\text { A: GGTGCTGACTGCATCGAAGGACAAA } \\
\text { S: CTGGCCTGCTGGCTCATGCTT }\end{array}$ & & \\
Actin & A: CCTCAGAAAGAGCAGCATCGATATG & 162 & NM_002427 \\
& S: CACCTTCTACAATGAGCTGCGTGT & \multirow{2}{*}{100} & NM_002046 \\
\hline
\end{tabular}

Table 2. Mankin Score System

\begin{tabular}{ll}
\hline Items & Score \\
\hline 1. Structure integrity & \\
Intact & 0 \\
Surface irregularity & 1 \\
Irregular surface plus pannus & 2 \\
Fissures to middle zone & 3 \\
Fissures to deep zone & 4 \\
Fissures to subchondral zone & 5 \\
Totally disrupted & 6 \\
2. Chondrocytes & \\
Normal & 0 \\
Slight loss of chondrocytes clusters & 1 \\
25\% of the clusters & 2 \\
No clusters & 3 \\
3. Safranin-O staining & \\
Normal & \\
Slight loss & 0 \\
Moderate & 1 \\
Slight & 2 \\
None & \\
Intact & \\
\hline Vascular penetration & \\
\hline & \\
\hline
\end{tabular}

Table 3. Primers of targeted genes. $S=$ Sense; $\mathrm{A}=$ Antisense

\begin{tabular}{|c|c|c|c|}
\hline Targeted genes & Sequence $\left(5^{\prime}-3^{\prime}\right)^{*}$ & $\begin{array}{l}\text { Amplicon } \\
\text { length(bp) }\end{array}$ & Accession number \\
\hline MMP-1 & $\begin{array}{l}\text { S: ATGGCCTAGCAGTTGAGAAGCTGA } \\
\text { A: TCCACACCTGGGTTGCTTCATCAT }\end{array}$ & 110 & $\mathrm{AH} 005676$ \\
\hline MMP-3 & $\begin{array}{l}\text { S: ACACCGGATCTGCCAAGAGA } \\
\text { A: CTGGAGAACGTGAGTGGAGTCA }\end{array}$ & 89 & NM_001082280 \\
\hline MMP-13 & $\begin{array}{l}\text { S: CAGATGGGCATATCCCTCTAAGAA } \\
\text { A: CCATGACCAAATCTACAGTCCTCAC, }\end{array}$ & 88 & NM 001082037 \\
\hline 18SrRNA & $\begin{array}{l}\text { S: GACGGACCAGAGCGAAAGC } \\
\text { A: CGCCAGTCGGCATCGTTTATG }\end{array}$ & 119 & EU236696 \\
\hline
\end{tabular}




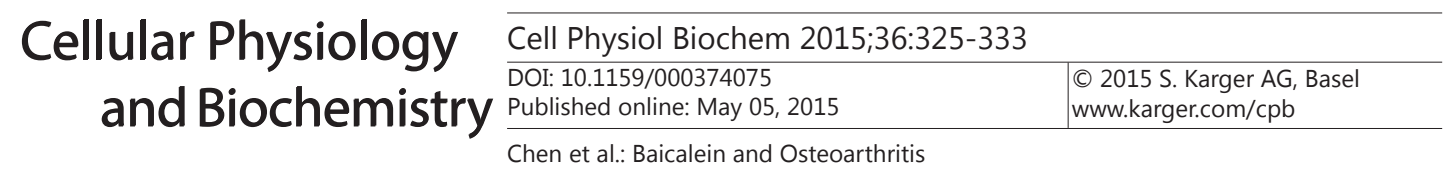

Detection gene expression in cartilage

Cartilage was collected and stored in liquid nitrogen for the detection of MMP-1, MMP-3 and MMP13 gene expression using quantitative real-time PCR as indicated above. Primer sequences of the targeted genes are listed in Table 3.

\section{Statistical analysis}

Data are expressed as means \pm standard deviation. Data were analyzed using a one way-ANOVA test. Dunnett-t method was used as the post-test in the ANOVA. A value of $\mathrm{P}<0.05$ was taken to indicate statistical significance

\section{Results}

Effect of baicalein on IL-1 $\beta$-induced MMPs expression in chondrocytes

The MTT assay demonstrated that $50 \mu \mathrm{M}$ baicalein had no adverse effects on cell viability (data not shown). Therefore, we investigated the effects of baicalein on IL-1 $\beta$ induced MMP expression in human chondrocytes. Baicalein treatment resulted in a dosedependent reduction in MMP-1, MMP-3 and MMP-13 gene expression (Fig. 1). In addition, baicalein reduced the levels of MMP-1, MMP-3 and MMP-13 in the culture media (Fig. 2).

Effects of baicalein on IL-1 $\beta$-induced mitogen-activated protein kinase (MAPK) activation in chondrocytes

To evaluate the underlying mechanisms responsible for the baicalein-mediated reduction of MMPs, we investigated the effects of baicalein on MAPK in IL-1 $\beta$-stimulated chondrocytes. IL-1 $\beta$ induced JNK, p38 and ERK phosphorylation, which were reduced by baicalein for p38 and ERK, whereas JNK was unaffected (Fig. 3).

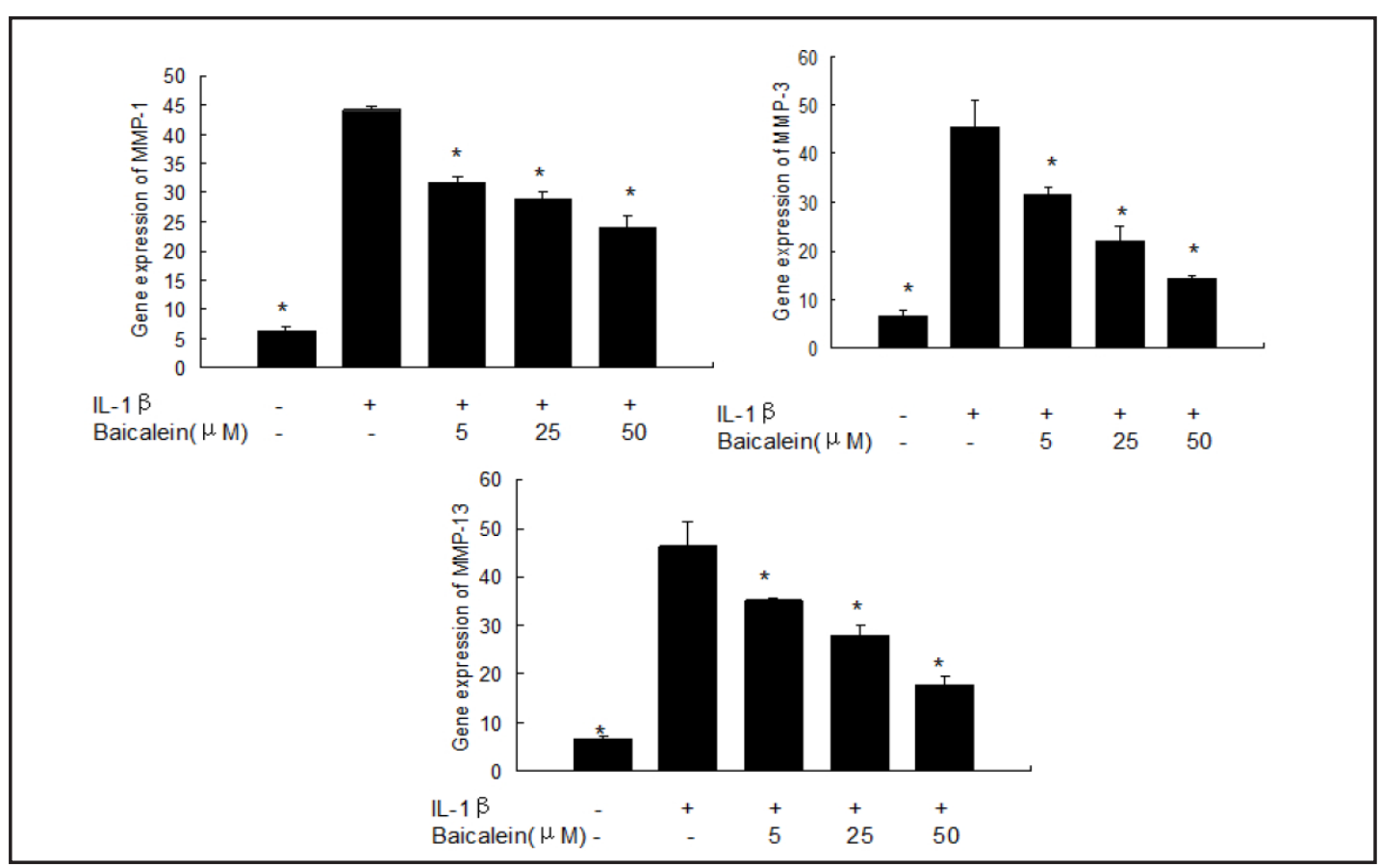

Fig. 1. Baicalein treatment inhibited interleukin (IL)-1 $\beta$-induced matrix metalloproteinases (MMPs) gene expression. Chondrocytes were pre-treated with various concentrations of baicalein for $1 \mathrm{~h}$, followed by stimulation with IL-1 $\beta(10 \mathrm{ng} / \mathrm{ml})$ for $24 \mathrm{~h}$. The chondrocytes were collected for gene expression analysis by PCR. Baicalein significantly inhibited MMP-1, MMP-3 and MMP-13 expression. Data are expressed as means \pm standard deviation (SD). ${ }^{*} \mathrm{P}<0.05$ compared to cells stimulated with IL-1 $\beta$ alone. 

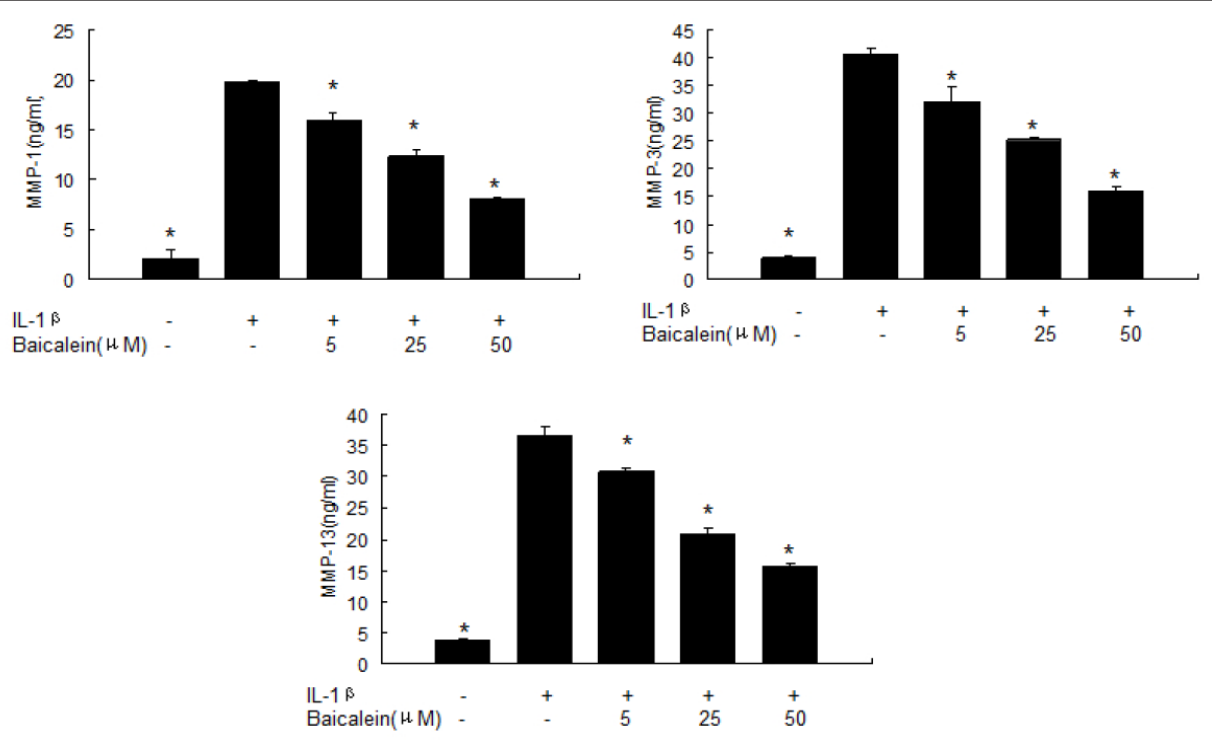

Fig. 2. Baicalein treatment inhibited interleukin (IL)-1ß-induced matrix metalloproteinases (MMPs) production. Chondrocytes were pre-treated with various concentrations of baicalein for $1 \mathrm{~h}$, followed by stimulation with IL-1 $\beta(10 \mathrm{ng} / \mathrm{ml})$ for $24 \mathrm{~h}$. The supernatants were collected and the concentrations of MMP-1, MMP-3 and MMP-13 were determined using an enzyme-linked immunosorbent assay. Baicalein significantly reduced the synthesis of MMP-1, MMP-3 and MMP-13. Data are expressed as means \pm standard deviation (SD). * $\mathrm{P}<0.05$ compared to cells stimulated with IL-1 $\beta$ alone.

Fig. 3. Baicalein inhibited interleukin (IL)-1 $\beta$-induced extracellular signal regulated kinase (ERK) and p38 but not c-Jun N-terminal kinase (JNK) activity. Chondrocytes were pretreated with baicalein for 24 $\mathrm{h}$, followed by stimulation with IL-1 $\beta(10 \mathrm{ng} / \mathrm{ml})$ for $30 \mathrm{~min}$. Western blotting was performed to evaluate phosphorylation of JNK, p38 and ERK. Baicalein inhibited IL-1 $\beta$-induced ERK and p38, but not JNK phosphorylation.

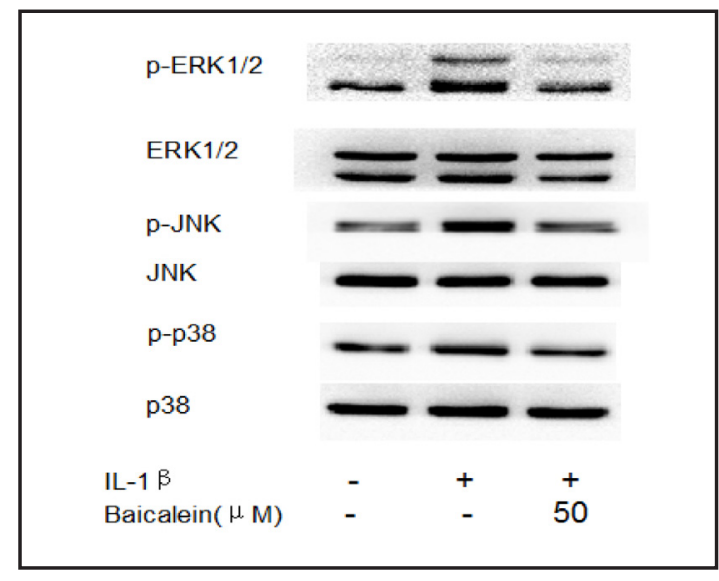

Fig. 4. Gross morphological grading. Rabbits received intra-articular injections of baicalein or vehicle once weekly for 6 weeks, initiated 1 day after the operation. There was no significant difference between OA group and baicalein-treated group in gra$\operatorname{ding}(\mathrm{P}>0.05)$.

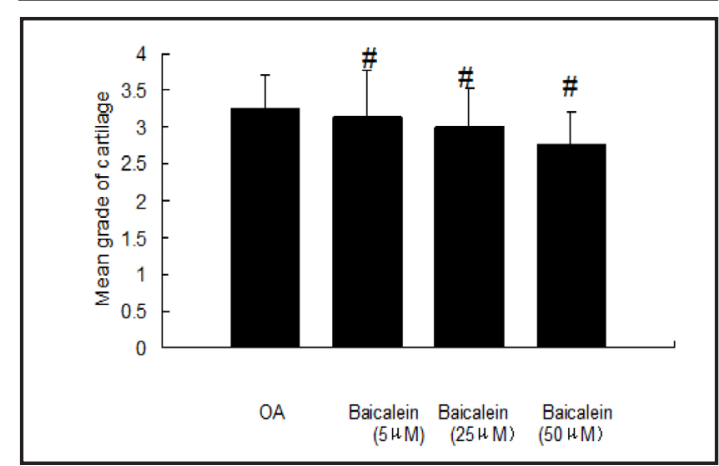

Gross morphological cartilage changes in a rabbit OA model

In the normal group, the articular cartilage was smooth and no lesions were observed on the surface. In contrast, in the OA group, the articular cartilage exhibited varying degrees of damage, the cartilage surface was rough and ulcers. In the baicalein-treated groups, the 


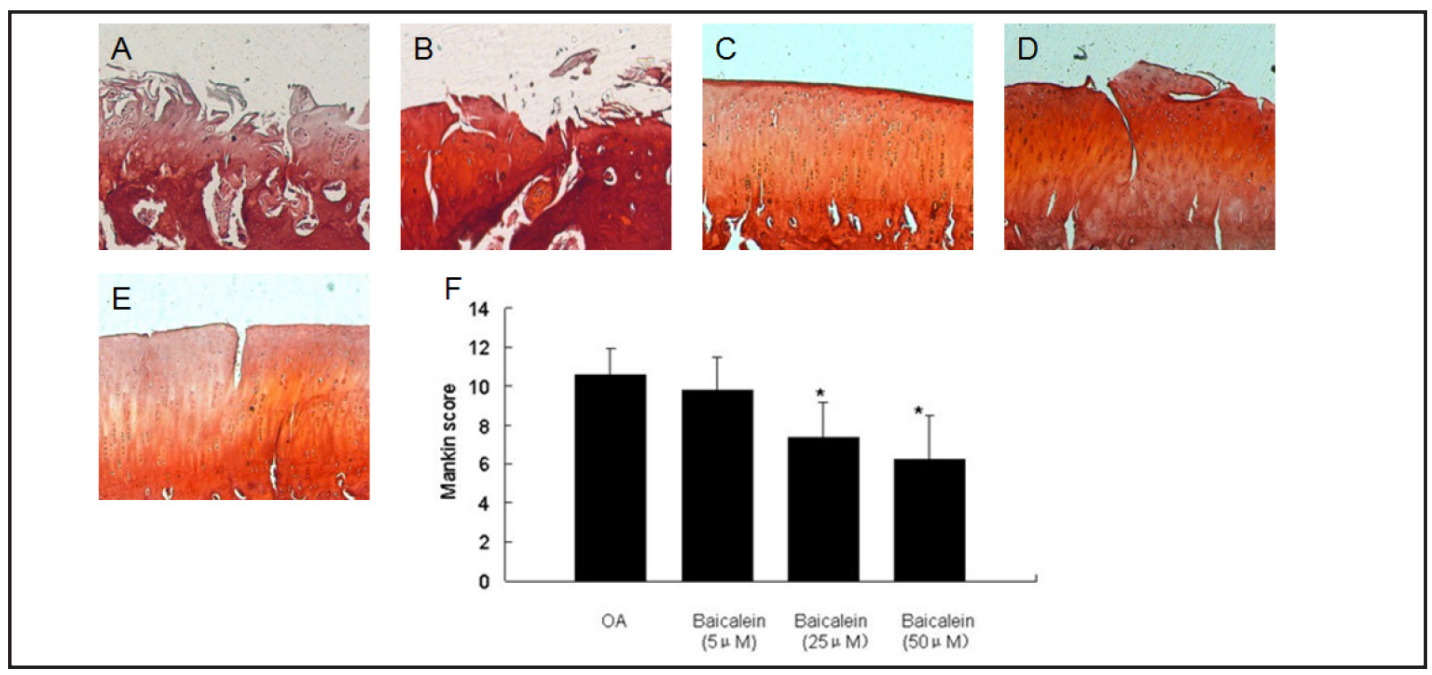

Fig. 5. Histological evaluation. Safranin-O staining was performed on cartilage sections. Typical changes in cartilage lesions are shown in. A: osteoarthritis (OA) group; B: baicalein ( $5 \mu \mathrm{M})$; C: normal; D: baicalein $(25 \mu \mathrm{M})$; E: baicalein $(50 \mu \mathrm{M})$. (Original magnification $\times 50$.); The Mankin scores were also assessed (F). The Mankin scores of medium and high-baicalein-concentration groups were lower than that of the osteoarthritis $(\mathrm{OA})$ group. ${ }^{*} \mathrm{P}<0.05$ when compared to cartilage from the $\mathrm{OA}$ group.

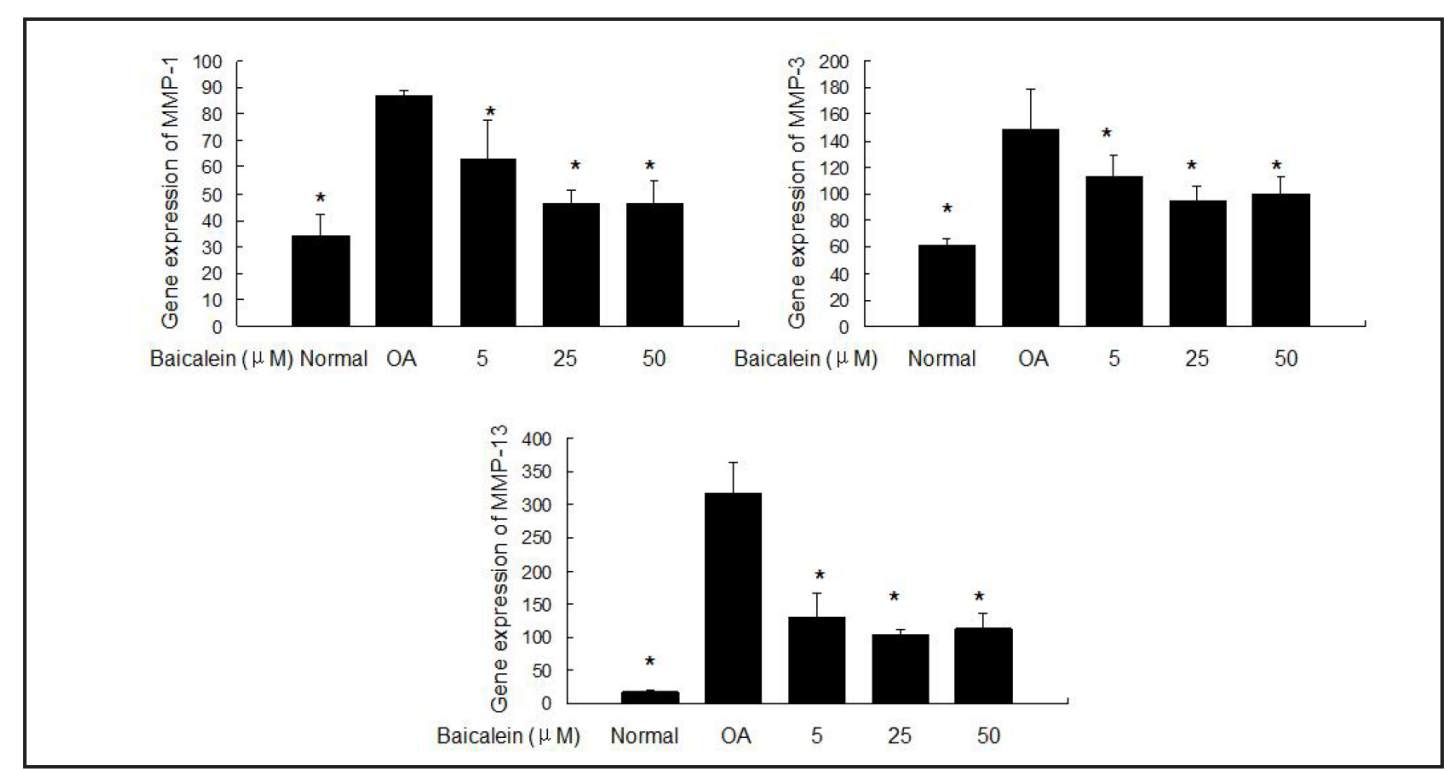

Fig. 6. Baicalein inhibited matrix metalloproteinases (MMPs) gene expression in cartilage. The expression of MMP-1, MMP-3 and MMP-13 in cartilage was analyzed using quantitative real-time polymerase chain reaction and normalized to that of $18 \mathrm{~S}$ rRNA. Baicalein inhibited the expression of MMP-1, MMP-3 and MMP13. Data are presented as means \pm standard deviation (SD). $* \mathrm{P}<0.05$ when compared to cartilage from the osteoarthritis (OA) group.

articular cartilage also showed lesions. And there was no significant difference between OA group and baicalein-treated group (Fig. 4).

\section{Histological evaluation}

The cartilage of the normal group had a normal histological appearance, whereas that of the OA group displayed clear hypocellularity and fissures to the deep zone. Cartilage from the low-concentration baicalein-treated group was similar to the OA group. Intra-articular injection of medium and high baicalein concentrations significantly ameliorated the cartilage 


\section{Cellular Physiology Cell Physiol Biochem 2015;36:325-333 \begin{tabular}{l|l} 
and Biochemistry Published online: May 05, 2015 & $\begin{array}{l}\text { C) 2015 S. Karger AG, Basel } \\
\text { www.karger.com/cpb }\end{array}$ \\
\hline
\end{tabular} \\ Chen et al.: Baicalein and Osteoarthritis}

damage compared to the OA group (Fig. 5). The Mankin scores for the cartilage are presented in Fig. 5.

\section{Cartilage gene expression}

MMP-1, MMP-3 and MMP-13 expression in the OA group was significantly increased compared to that in normal cartilage. Baicalein treatment reduced MMP-1, MMP-3 and MMP13 expression in a dose-dependent manner (Fig. 6).

\section{Discussion}

NSAIDs were used extensively in the treatment of OA to ameliorate pain. However, longterm use of NSAID can lead to serious side effects, particularly in the gastrointestinal and cardiovascular systems. Therefore, a new, safer agent which can ameliorate pain is required for OA. Phytochemicals are potential candidates because of their safety for long-term use.

Scutellaria baicalensis Georgi (Huangqin) is a herb with anti-inflammatory properties which was used widely for many centuries in China. Baicalein, a flavonoid isolated from Scutellaria baicalensis Georgi (Huangqin), is reported to possess multiple biological activities. In the present study, we evaluated its anti-osteoarthritic properties in vitro and in vivo. Baicalein inhibited the expression of key molecules, including MMP-1, MMP-3 and MMP-13 in IL-1 $\beta$ stimulated articular chondrocytes. This anti-osteoarthritic effect of baicalein was due in part to its inhibition of the MAPK pathway. In vivo, we found that baicalein significantly reduced cartilage damage as well as MMP-1, MMP-3 and MMP-13 expression in cartilage. Therefore, the findings were consistent in both models: in vitro in IL-1 $\beta$-induced chondrocytes and in an ACLT-induced OA rabbit model.

MMPs is a family of enzymes responsible for ECM degradation. Previous studies demonstrated that MMP-1, MMP-3 and MMP-13 play important roles in OA. In particular, MMP-13 is considered a potent protease for cartilage damage because of its ability to breakdown type II collagen, the main ECM component. Therefore, inhibiting MMP activities would be expected to ameliorate OA progression. In the present study, we demonstrated that baicalein effectively suppressed the overexpression of MMP-1, MMP-3 and MMP-13 in IL-1 $\beta$ stimulated chondrocytes. Previous studies showed that baicalein reduced MMP-2 and MMP9 expression in glioma and hepatocellular carcinoma cells $[18,19]$. In addition, MMP-1 was inhibited by baicalein in human keratinocytes [11]. Moreover, Zhang et al. reported recently that baicalein inhibited MMP-3 and MMP-13 expression in chondrocytes stimulated using a mixture of IL-1 $\beta$ and tumor necrosis factor- $\alpha$ (TNF- $\alpha$ ) [20]. Therefore, we hypothesized that baicalein may exert beneficial effects in OA via reducing MMPs activities and expression.

In the present study, we also investigated the mechanisms underlying the inhibition by baicalein of IL-1 $\beta$-induced MMP expression in chondrocytes. Many signaling pathways are known to be involved in cartilage degradation in OA; Of these, we focused on the MAPK signaling pathway because of its importance in OA progression. The MAPK signaling pathway is involved in MMPs regulation [21]. For example, the p38 signaling pathway was reported to induce MMPs expression, leading to ECM degradation [22]. In the present study, we demonstrated that IL-1 $\beta$ stimulation induced the phosphorylation of p38, ERK and JNK, while baicalein reduced the phosphorylation of p38 and ERK, whereas there was no significant inhibition of phospho-JNK formation. Our findings are at least in part consistent with previous reports using other cell types. Zhang et al. demonstrated that baicalein inhibited p38 activation in glioma cells, whereas ERK and JNK were unaffected [23]. Liu et al. found that baicalein inhibited p38 activation in human melanocytes, whereas ERK activation was unaffected [24]. In a further study, Chen et al. reported that baicalein exerted its effect via down-regulation of the ERK pathway in hepatocellular carcinoma cells [25]. These studies indicate that baicalein can affect the activation of MAPK. The reasons for the discrepancies among the studies may be use of different cell types and/or experimental conditions. Therefore, we hypothesized that baicalein inhibits MMPs expression in chondrocytes at least in part through the inhibition of p38 and ERK activation. However, the exact 


\begin{tabular}{|c|c|c|}
\hline Cellular Physiology & Cell Physiol Biochem 2015;36:325-333 & \\
\hline and Biochemistry & $\begin{array}{l}\text { DOI: 10.1159/000374075 } \\
\text { Published online: May 05, } 2015\end{array}$ & $\begin{array}{l}\text { O } 2015 \text { S. Karger AG, Basel } \\
\text { www.karger.com/cpb }\end{array}$ \\
\hline
\end{tabular}

mechanism associated with baicalein andMAPK is still unclear, it is still unknown whether ERK and p38 are direct targets of baicalein, thus, further studies are needed to explore the underlying mechanism by which baicalein regulates MAPK signaling pathway. In addition, the mechanism underlying the regulation of MMPs in chondrocytes by baicalein remains unclear. Further studies are required to elucidate the mechanism of the anti-osteoarthritic effects of baicalein.

We investigated the in vivo effects of baicalein on cartilage in experimental OA over a period of 6 weeks, which is similar to the durations used in previous studies [26, 27]. We found that the cartilage displayed OA-like changes; Therefore, 6 weeks was sufficient to induce OA using ACLT. However, a longer study may provide more information regarding the effects of baicalein on cartilage damage in experimental OA. Indeed, some studies investigated three stages of ACLT-induced OA for up to 12 weeks $[28,29]$. Therefore, further studies are required to obtain a better understanding of the in vivo effects of baicalein on cartilage damage in $\mathrm{OA}$.

In conclusion, we demonstrated that baicalein inhibits MMPs expression in chondrocytes, which is mediated at least in part by the suppression of the activation of the p38 and ERK signaling pathways. Moreover, intra-articular injections of baicalein in experimental OA reduced cartilage damage. These findings indicate a potential therapeutic role for baicalein in $\mathrm{OA}$.

\section{Acknowledgements}

This study was supported by the National Natural Science Foundation of China (81201429)

\section{Disclosure Statement}

The authors declare that there are no conflicts of interest.

\section{References}

1 Burrage PS, Mix KS, Brinckerhoff CE: Matrix metalloproteinases: Role in arthritis. Front Biosci 2006;11:529-543.

2 Stove J, Huch K, Gunther KP, Scharf HP: Interleukin-1beta induces different gene expression of stromelysin, aggrecan andtumor-necrosis-factor-stimulated gene 6 in human osteoarthritic chondrocytes in vitro. Pathobiology 2000;68:144-149.

3 Neuhold LA, Killar L, Zhao W, Sung ML, Warner L, Kulik J, Turner J, Wu W, Billinghurst C, Meijers T, Poole AR, Babij P, DeGennaro LJ: Postnatal expression in hyaline cartilage of constitutively active human collagenase-3 (MMP-13) induces osteoarthritis in mice. J Clin Invest 2001;107:35-44.

4 Mengshol JA, Vincenti MP, Coon CI, Barchowsky A, Brinckerhoff CE: Interleukin-1 induction of collagenase 3 (matrix metalloproteinase 13) gene expression in chondrocytes requires p38, c-Jun N-terminal kinase, and nuclear factor kappaB: Differential regulation of collagenase 1 and collagenase 3 . Arthritis Rheum 2000;43:801-811.

5 Sabatini M, Lesur C, Thomas M, Chomel A, Anract P, de Nanteuil G, Pastoureau P: Effect of inhibition of matrix metalloproteinases on cartilage loss in vitro andin a guinea pig model of osteoarthritis. Arthritis Rheum 2005;52:171-180.

6 Kobayashi M, Squires GR, Mousa A, Tanzer M, Zukor DJ, Antoniou J, Feige U, Poole AR: Role of interleukin-1 and tumor necrosis factor alpha in matrix degradation of human osteoarthritic cartilage. Arthritis Rheum 2005;52:128-135.

7 Fan GW, Zhang Y, Jiang X, Zhu Y, Wang B, Su L, Cao W, Zhang H, Gao X: Anti-inflammatory activity of baicalein in LPS-stimulated RAW264.7 macrophages via estrogen receptor and NF-kappaB-dependent pathways. Inflammation 2013;36:1584-1591.

8 Qi Z, Yin F, Lu L, Shen L, Qi S, Lan L, Luo L, Yin Z: Baicalein reduces lipopolysaccharide-induced inflammation via suppressing JAK/STATs activation and ROS production. Inflamm Res 2013;62:845-855. 


\section{Cellular Physiology Cell Physiol Biochem 2015;36:325-333 \begin{tabular}{l|l|l} 
DOI: 10.1159/000374075 & (C) 2015 S. Karger AG, Basel
\end{tabular} and Biochemistry Published online: May 05, $2015 \quad$ www.karger.com/cpb \\ Chen et al.: Baicalein and Osteoarthritis}

9 Kim KC, Lee IK, Kang KA, Kim HS, Kang SS, Hyun JW: Baicalein (5,6,7-trihydroxyflavone) reduces oxidative stress-induced DNA damage by upregulating the DNA repair system. Cell Biol Toxicol 2012;28:421-433.

10 Zheng YH, Yin LH, Grahn TH, Ye AF, Zhao YR, Zhang QY: Anticancer Effects of Baicalein on Hepatocellular Carcinoma Cells. Phytother Res 2014

11 Kim KC, Kang SS, Lee J, Park D, Hyun JW: Baicalein attenuates oxidative Stress-Induced expression of matrix metalloproteinase-1 by regulating the ERK/JNK/AP-1 pathway in human keratinocytes. Biomol Ther (Seoul) 2012;20:57-61.

12 Chen S, Yang Y, Feng H, Wang H, Zhao R, Liu H: Baicalein inhibits interleukin-1beta-induced proliferation of human rheumatoid arthritis fibroblast-like synoviocytes. Inflammation 2014;37:163-169.

13 Chen WP, Xiong Y, Shi YX, Hu PF, Bao JP, Wu LD: Astaxanthin reduces matrix metalloproteinase expression in human chondrocytes. Int Immunopharmacol 2014;19:174-177.

14 Chen WP, Wang YL, Tang JL, Hu PF, Bao JP, Wu LD: Morin inhibits interleukin-1beta-induced nitric oxide and prostaglandin E2 production in human chondrocytes. Int Immunopharmacol 2012;12:447-452.

15 Chen WP, Bao JP, Hu PF, Feng J, Wu LD: Alleviation of osteoarthritis by Trichostatin a, a histone deacetylase inhibitor, in experimental osteoarthritis. Mol Biol Rep 2010;37:3967-3972.

16 Shikhman AR, Amiel D, D'Lima D, Hwang SB, Hu C, Xu A, Hashimoto S, Kobayashi K, Sasho T, Lotz MK: Chondroprotective activity of $\mathrm{N}$-acetylglucosamine in rabbits with experimental osteoarthritis. Ann Rheum Dis 2005;64:89-94.

17 Mankin HJ, Dorfman H, Lippiello L, Zarins A: Biochemical and metabolic abnormalities in articular cartilage from osteo-arthritic human hips. II. Correlation of morphology with biochemical and metabolic data. J Bone Joint Surg Am 1971;53:523-537.

18 Zhang Z, Lv J, Lei X, Li S, Zhang Y, Meng L, Xue R, Li Z: Baicalein reduces the invasion of glioma cells via reducing the activity of p38 signaling pathway. PLoS One 2014;9:e90318.

19 Chiu YW, Lin TH, Huang WS, Teng CY, Liou YS, Kuo WH, Lin WL, Huang HI, Tung JN, Huang CY, Liu JY, Wang WH, Hwang JM, Kuo HC: Baicalein inhibits the migration and invasive properties of human hepatoma cells. Toxicol Appl Pharmacol 2011;255:316-326.

20 Zhang X, Zhu Y, Chen X, Zhang Y, Zhang Y, Jia Y, Wang H, Liu Y, Xiao L: Baicalein ameliorates inflammatoryrelated apoptotic and catabolic phenotypes inhuman chondrocytes. Int Immunopharmacol 2014;21:301308.

21 Yan C, Boyd DD: Regulation of matrix metalloproteinase gene expression. J Cell Physiol 2007;211:19-26.

22 Acha O, Hernandez JL, Penado S, Cano M, Riancho JA: [Risk factors and stroke among patients of different ages]. Rev Clin Esp 2003;203:189-192.

23 Zhang Z, Lv J, Lei X, Li S, Zhang Y, Meng L, Xue R, Li Z: Baicalein reduces the invasion of glioma cells via reducing the activity of p38 signaling pathway. PLoS One 2014;9:e90318.

24 Liu B, Jian Z, Li Q, Li K, Wang Z, Liu L, Tang L, Yi X, Wang H, Li C, Gao T: Baicalein protects human melanocytes from $\mathrm{H}(2) \mathrm{O}(2)$-induced apoptosis via inhibiting mitochondria-dependent caspase activation and the p38 MAPK pathway. Free Radic Biol Med 2012;53:183-193.

25 Chen K, Zhang S, Ji Y, Li J, An P, Ren H, Liang R, Yang J, Li Z: Baicalein inhibits the invasion and metastatic capabilities of hepatocellular carcinoma cells via down-regulation of the ERK pathway. PLoS One 2013;8:e72927.

26 Akasaki Y, Matsuda S, Nakayama K, Fukagawa S, Miura H, Iwamoto Y: Mevastatin reduces cartilage degradation in rabbit experimental osteoarthritis through inhibition of synovial inflammation. Osteoarthritis Cartilage 2009;17:235-243.

27 Chen WP, Tang JL, Bao JP, Hu PF, Shi ZL, Wu LD: Anti-arthritic effects of chlorogenic acid in interleukin1beta-induced rabbit chondrocytes and a rabbit osteoarthritis model. Int Immunopharmacol 2011;11:2328.

28 Mehraban F, Tindal MH, Proffitt MM, Moskowitz RW: Temporal pattern of cysteine endopeptidase (cathepsin B) expression in cartilageand synovium from rabbit knees with experimental osteoarthritis: Gene expressionin chondrocytes in response to interleukin-1 and matrix depletion. Ann Rheum Dis 1997;56:108-115.

29 Bao JP, Chen WP, Feng J, Zhao J, Shi ZL, Huang K, Wu LD: Variation patterns of two degradation enzyme systems in articular cartilage in different stages of osteoarthritis: Regulation by dehydroepiandrosterone. Clin Chim Acta 2009;408:1-7. 